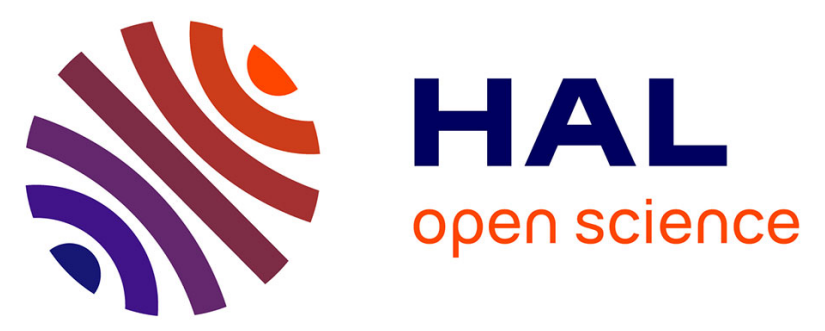

\title{
Blind Adaptive Transmitter IQ Imbalance Compensation in M-QAM Optical Coherent Systems
}

Trung Hien Nguyen, Pascal Scalart, Mathilde Gay, Laurent Bramerie, Christophe Peucheret, Ti Nguyen-Ti, Matthieu Gautier, Olivier Sentieys, Jean-Claude Simon, Michel Joindot

\section{To cite this version:}

Trung Hien Nguyen, Pascal Scalart, Mathilde Gay, Laurent Bramerie, Christophe Peucheret, et al.. Blind Adaptive Transmitter IQ Imbalance Compensation in M-QAM Optical Coherent Systems. 2016 IEEE International Conference on Communication (ICC 2016), May 2016, Kuala Lumpur, Malaysia. 10.1109/ICC.2016.7510925 . hal-01337225

\section{HAL Id: hal-01337225 https://hal.science/hal-01337225}

Submitted on 24 Jun 2016

HAL is a multi-disciplinary open access archive for the deposit and dissemination of scientific research documents, whether they are published or not. The documents may come from teaching and research institutions in France or abroad, or from public or private research centers.
L'archive ouverte pluridisciplinaire $\mathbf{H A L}$, est destinée au dépôt et à la diffusion de documents scientifiques de niveau recherche, publiés ou non, émanant des établissements d'enseignement et de recherche français ou étrangers, des laboratoires publics ou privés. 


\title{
Blind Adaptive Transmitter IQ Imbalance Compensation in $M$-QAM Optical Coherent Systems
}

\author{
Trung-Hien Nguyen ${ }^{1}$, Pascal Scalart ${ }^{2}$, Mathilde Gay ${ }^{1}$, Laurent Bramerie ${ }^{1}$, Christophe Peucheret ${ }^{1}$, Ti Nguyen-Ti ${ }^{2}$, \\ Matthieu Gautier ${ }^{2}$, Olivier Sentieys ${ }^{2}$, Jean-Claude Simon ${ }^{1}$ and Michel Joindot ${ }^{1}$ \\ ${ }^{1}$ FOTON Laboratory, CNRS, University of Rennes 1, ENSSAT, F-22305 Lannion, France \\ ${ }^{2}$ INRIA / IRISA, University of Rennes 1, 35000 Rennes, France \\ trung-hien.nguyen@enssat.fr
}

\begin{abstract}
Blind adaptive source separation (BASS) based compensation for transmitter (Tx) IQ imbalance is presented for the first time in an $M$-QAM optical coherent system. The proposed method is numerically investigated with 4-QAM and 16-QAM signals in the presence of $T x I Q$ imbalance up to $30^{\circ}$. The robustness of the BASS method is studied after $200-\mathrm{km}$ optical fiber transmission, in which the effects of chromatic dispersion (CD) and carrier frequency offset (CFO) are assumed to be dominant. It is also found that CFO, inherent to frequency difference between the transmitter and receiver lasers in optical coherent transmission, should be compensated before IQ imbalance compensation to achieve a better performance. The proposed method outperforms the Gram-Schmidt orthogonalization procedure (GSOP) in the presence of CD and CFO. We further validate experimentally the proposed method with 10-Gbaud optical 4-QAM and 16-QAM signals at $30^{\circ}$ and $10^{\circ}$ phase imbalance, respectively, with an emulated $200-\mathrm{km}$ optical fiber transmission and 200-MHz CFO. More specifically, the optical signal-to-noise ratio (OSNR) penalty reduction of the BASS method compared to the GSOP method is $1 \mathrm{~dB}$ for 4-QAM at a bit-error-ratio (BER) of $2 \times 10^{-3}$ and $2 \mathrm{~dB}$ for 16-QAM at a BER of $10^{-3}$. Moreover, instead of being a fully independent block and requiring statistical estimation as in GSOP, the BASS method can be integrated into an equalizer and operated at the sample rate, simplifying the operation and allowing parallel implementation.
\end{abstract}

Keywords-IQ imbalance compensation; M-QAM; optical coherent transmission; digital signal processing

\section{INTRODUCTION}

$M$-ary quadrature amplitude modulation ( $M$-QAM, $M>4)$ combined with coherent detection and digital signal processing (DSP) becomes now a promising candidate for the implementation of next generation optical transmission systems. This is made possible notably thanks to technical progress in photonic integrated circuits (PICs) allowing the fabrication of monolithically integrated optical circuits for $M$-QAM optical signal generation [1]. Despite the amazing performance of these circuits, there are still some issues, in particular concerning the nonlinear gain of electrical amplifiers, the control of phase shifts in optical waveguides and cable lengths or circuit paths on printed boards. For all these reasons the resulting signal may present amplitude imbalance, angular imbalance, or timing skew, globally referred to as IQ imbalance.

As the number of modulation levels increases, the sensitivity to such imperfections is exacerbated, especially under the impact of carrier frequency offset (CFO) between the transmitter (Tx) laser and receiver $(\mathrm{Rx})$ and chromatic dispersion (CD). Some effort has been dedicated to Rx IQ imbalance compensation with the help of DSP, including the Gram-Schmidt orthogonalization procedure (GSOP) [2] or complex-valued multiple-input multi-output (MIMO) adaptive equalizers [3]. Tx IQ imbalance is more critical in transmission systems and some recent work has attempted to eliminate this impairment, e.g. by using Turbo demodulation of LDPC-coded signals [4], or indirect learning architecture [5] as a pre-compensation, regardless of transmission imperfections.

In this paper, we propose a Tx IQ imbalance compensation method based on a blind adaptive source separation (BASS) algorithm [6] cascaded to a real-valued MIMO equalizer. In this approach, we demonstrate simultaneous IQ imbalance and $\mathrm{CD}$ compensation. Note that the proposed algorithm is also suitable for Rx IQ imbalance compensation and for both single and multi carrier advanced modulation format signals, which is a desirable feature in the context of flexible optical transceivers [7]. Since the GSOP is widely used in optical fiber communications [8], it is utilized here as a benchmark to compare with our proposed method. To the best of our knowledge, the GSOP method is also studied here for the first time to compensate for Tx IQ imbalance of a single carrier optical $M$-QAM signal after 200-km transmission, in which $\mathrm{CD}$ is the dominant signal degradation. Performance comparison between the GSOP and the BASS method is first carried out numerically with 4-QAM (also known as quadrature phase shift keying - QPSK) and 16-QAM signals. In the presence of a strong Tx IQ imbalance (i.e. $20^{\circ}$ phase imbalance), it is found that CFO should be compensated for before IQ imbalance, regardless of the used compensation method. The results show that our proposed method is more robust against residual $\mathrm{CFO}$ and $\mathrm{CD}$ induced by optical fiber transmission compared to the GSOP. An experimental validation is also carried out with 10-Gbaud optical 4-QAM 
and 16-QAM signals subjected to $30^{\circ}$ and $10^{\circ}$ phase imbalance, respectively. Our proposed method enables optical signal-to-noise ratio (OSNR) penalty reductions of $1 \mathrm{~dB}$ and $2 \mathrm{~dB}$ at bit-error-ratios (BERs) of $2 \times 10^{-3}$ and $10^{-3}$ for 4-QAM and 16-QAM signals, respectively, compared to the GSOP method, showing its effectiveness. Furthermore, while the GSOP method operates based on statistical calculations on collected samples, our proposed method effectively operates at the sample rate, possibly facilitating parallel implementations, hence is promising for high bit-rate transmission systems.

The paper is organized as follows: Section II briefly describes a model for Tx IQ imbalance under the impacts of CFO and CD. Numerical studies of the proposed method are then reported in Section III. The effectiveness of the proposed method is further validated experimentally in Section IV. A comparison of hardware complexity of the algorithms is summarized in Section V. Finally, Section VI concludes the paper.

\section{TX IQ IMBALANCE UNDER THE IMPACTS OF CFO AND CD}

Fig. 1 presents a block diagram of an $M$-QAM coherent transmission system. The different driving electrical signal amplitudes between the in-phase (I) and quadrature (Q) arms of the modulator and the imperfect biasing are referred to as Tx loss imbalance, $\varepsilon$, and phase imbalance, $\theta$, respectively [4]. Due to the symmetry of the I and Q components, we assume $0 \leq \theta \leq \pi / 2$ and $0 \leq \varepsilon \leq 1$. Under the impact of Tx IQ imbalance, the transmitted signal, $x(t)$, can be expressed as [9]

$$
\begin{aligned}
& x(t)=\mathfrak{R}\left[s(t) \cdot\left(G_{1} e^{j \omega_{s} t}+G_{2} e^{-j \omega_{s} t}\right)\right] \\
& G_{1}=\left(1+\varepsilon e^{j \theta}\right) / 2, \quad G_{2}=\left(1-\varepsilon e^{-j \theta}\right) / 2
\end{aligned}
$$

where $s(t)$ and $\omega_{S}$ denote the baseband signal to be transmitted and the Tx laser frequency, respectively. $(\cdot)^{*}$ is the complex conjugation operation, $\mathfrak{R}$ is the real part operator and $j=\sqrt{-1}$. It should be noted that $G_{1}=1$ and $G_{2}=0$ in the absence of Tx IQ imbalance. This distorted signal is then transmitted over a $200-\mathrm{km}$ optical fiber. The system is considered as a linear time invariant (LTI) system in the absence of fiber nonlinearity. The resulting transfer function associated with $\mathrm{CD}, F(z, \Delta \omega)$, can be expressed as [10]

$$
F(z, \Delta \omega)=\exp \left(-j \frac{D \lambda^{2} z}{4 \pi c} \Delta \omega^{2}\right)
$$

where $z$ is the propagation distance. $\lambda$ and $c$ denote the wavelength and speed of the light in vacuum, respectively. $D$ represents the dispersion coefficient of the fiber. $\Delta \omega$ is the angular frequency with respect to $\omega_{S}$. Using this transfer function, the effect of $C D$ can be simulated so that simultaneous compensation of IQ imbalance and CD can be verified with the proposed method.

After being combined with the signal emitted by a local oscillator laser, $x_{L O}(t)$, in a coherent receiver and experiencing the effect of CFO, $\omega_{0}=\omega_{L}-\omega_{S}\left(\omega_{L}\right.$ being the Rx laser angular frequency), the received I and Q components after balanced photodetection, can be written

$$
\begin{aligned}
& I=\mathfrak{R}\left(y \cdot x_{L O}^{*} \cdot e^{-j \omega_{0} t}\right) \\
& Q=\mathfrak{I}\left(y \cdot x_{L O}^{*} \cdot e^{-j \omega_{0} t}\right)
\end{aligned}
$$

in which $y(t)$ is the transmitted signal having experienced CD and $\mathfrak{I}$ is the imaginary part operator.

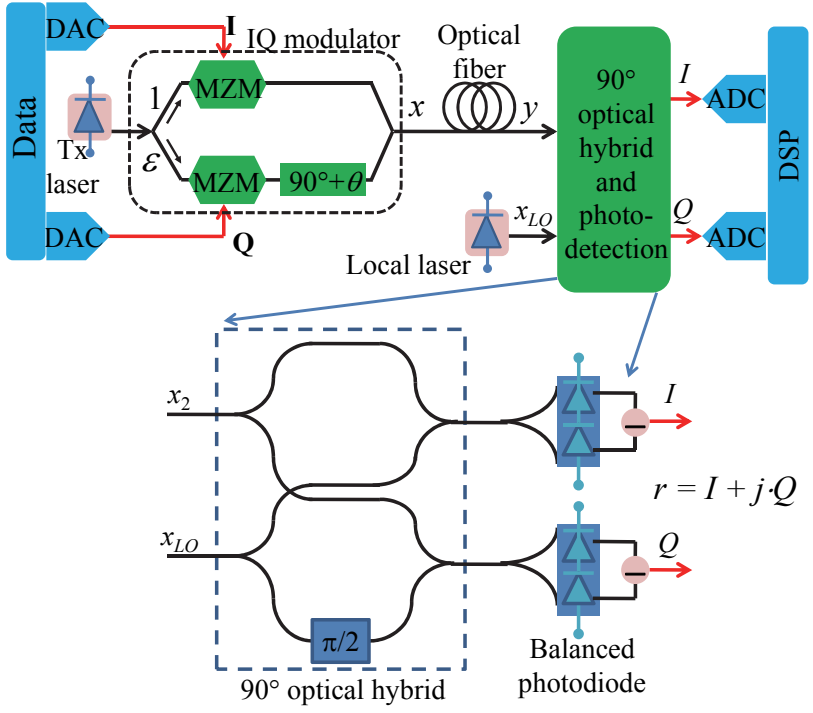

Fig. 1. Block diagram of the $M$-QAM coherent system under the impacts of Tx IQ imbalance, CFO and CD.

After digitizing the signals at the outputs of the balanced photodiodes using analog-to-digital converters (ADCs), the DSP steps are as follows [8]: (i) IQ imbalance compensation; (ii) $\mathrm{CD}$ compensation in the frequency domain [10]; (iii) retiming and resampling; (iv) frequency-domain based CFO compensation [11], (v) phase noise estimation [12] and (vi) bit-error-ratio (BER) calculation.

Concerning the Tx IQ imbalance compensation step, GSOP and BASS methods are compared. In fact, IQ imbalance results in an interfering term on the demodulated complex signal that is a linear combination of the useful signal and its complex conjugate version. This relation can be expressed as a matrix [13] and the compensation problem is equivalent to finding the best estimation of the inverse of this matrix in order to recover the useful signal by cancelling the interference. This becomes feasible due to the circular property of the signal constellation and due to the fact that $r(k)$ and $r^{*}(k)$, in which $r(k)=I+j \cdot Q$, are not correlated so that the statistical expectation $E\left(r^{2}\right)=0$ holds [13]. The problem now reduces to the whitening transformation [6] of the received samples blocks and their complex conjugates, which is the key idea behind the BASS method, so that the complex conjugation (induced by IQ imbalance) is eliminated. More specifically, we only need to compute 
$r^{\prime}(k)=w_{1}(k) r(k)+w_{2}(k) r^{*}(k)$, with $w_{1}$ and $w_{2}$ being adaptively deduced by [14]

$$
\begin{aligned}
& w_{1}(k+1)=\left[1-\mu\left(\left|r^{\prime}(k)\right|^{2}-1\right)\right] w_{1}(k)-\mu\left(r^{\prime}(k)\right)^{2} w_{2}^{*}(k) \\
& w_{2}(k+1)=\left[1-\mu\left(\left|r^{\prime}(k)\right|^{2}-1\right)\right] w_{2}(k)-\mu\left(r^{\prime}(k)\right)^{2} w_{1}^{*}(k)
\end{aligned}
$$

where $\mu$ is the step-size and $k$ denotes the index of the $k$-th sample. Note that, our algorithm is also modified to be compatible with fractionally-spaced equalizers, which further differentiates our study from the work in [14] that uses symbol-spaced equalizers. Note that, only one polarization is considered in this study. However, the proposed schemes can possibly be extended to polarization multiplexed signals. In the dual-polarization case, this algorithm may be integrated into the 1-tap constant modulus algorithm (CMA) based equalizer for simultaneous IQ imbalance compensation and polarization demultiplexing. This integration potentially offers reduced complexity compared to the IQ imbalance compensation method in [15]. Further discussion on polarization diversity receivers is out of the scope of this paper.

Because the loss imbalance could be compensated for by hardware implementations (such as the use of automatic gain controlled transimpedance amplifiers - TIAs) [16] at the receiver side, it is therefore not considered in this study for the sake of simplification. In the following parts, we numerically and experimentally investigate the performance of the GSOP and BASS methods in different cases:

- Case 1: back-to-back (B2B), corresponding to the case when the transmitter is directly connected to the receiver, without (w/o) CFO and CD impacts.

- Case 2: 200-km transmission (equivalent to an accumulated CD of $3400 \mathrm{ps} / \mathrm{nm}$ ) [17] with (w/) CFO compensation before IQ imbalance compensation (i.e. DSP step (iv) before step (i))

- Case 3: 200-km transmission with CFO compensation after IQ imbalance compensation (i.e. DSP step (iv) after step (i)).

\section{NUMERICAL INVESTIGATION}

To evaluate the performance of the proposed method, the setup in Fig. 1 is numerically simulated for 4-QAM and 16-QAM transmission systems. At the Tx, two pseudorandom binary sequences (PRBSs) with lengths of $2^{23}-1$ and $2^{15}-1$ bits, are used to generate the $10-\mathrm{Gbit} / \mathrm{s}$ data streams applied to the IQ modulator, resulting in a 10-Gbaud 4-QAM signal. For the generation of the 16-QAM signal, two 4-pulse amplitude modulation (PAM) sequences are constituted from two PRBSs with lengths of $2^{11}-1$ and $2^{13}-1$ bits. Those sequences are then applied to the $\mathrm{I}$ and $\mathrm{Q}$ inputs of the modulator. To focus on the impact of CFO and CD induced by transmission on the Tx IQ imbalance compensation, other nonlinear transmission effects are ignored. At the $\mathrm{Rx}$ the local laser frequency is set to obtain a $\mathrm{CFO}$ of $0.2 \mathrm{GHz}$ (to match typical experimental conditions). The resolution of the analogto-digital converters (ADCs) is assumed to be sufficiently high to neglect amplitude errors linked to quantization steps. Twenty calculation iterations (with different random noise seeds) are carried out for each IQ imbalance value before averaging the BER calculations over about 130000 symbols.

Fig. 2 shows the OSNR penalty for 4-QAM calculated at a BER of $10^{-3}$ as a function of phase imbalance. The results are obtained w/o IQ imbalance compensation and w/ the GSOP and BASS methods. The OSNR penalty increases with the increase of the phase imbalance. At $30^{\circ}$ phase imbalance, the penalties are $2.3 \mathrm{~dB}$ and $1 \mathrm{~dB}$ in case $1 \mathrm{w} / \mathrm{o}$ and $\mathrm{w} / \mathrm{IQ}$ imbalance compensation, respectively. The BASS method is slightly better than the GSOP method. In case 2 , the distorted signal is launched in a $200-\mathrm{km}$ fiber link and suffers from the impact of CFO at the Rx side. It can be seen that the penalty is increased both w/o and w/ IQ imbalance compensation. With compensation, an OSNR penalty inferior to $2 \mathrm{~dB}$ at $30^{\circ}$ phase imbalance can be achieved, while the OSNR penalty can reach $3.5 \mathrm{~dB}$ if no compensation is applied.

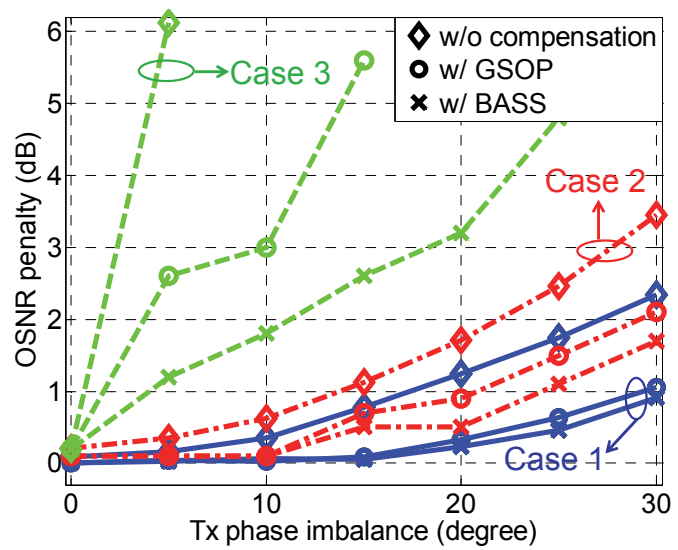

Fig. 2. OSNR penalty of 4-QAM versus Tx phase imbalance. Solid lines: case 1; Dash-dotted lines: case 2; Dashed lines: case 3.

In case 3 , the OSNR penalty is sharply increased even with small IQ imbalance (i.e. $10^{\circ}$ phase imbalance) regardless of the IQ imbalance compensation. The difference between cases 2 and 3 could come from the incorrect estimation of parameters for the GSOP and BASS methods due to the fast rotation effect of CFO. It is therefore necessary to remove the CFO impact before carrying out Tx IQ imbalance compensation. For this reason, the following studies consider only cases 1 and 2 . Note that, in all cases, the BASS method shows a better performance than the GSOP method.

Fig. 3 shows the results of the same study in the case of 16-QAM signals. Similar behaviors are also observed. However, the Tx phase imbalance effect is more critical, as indicated by the larger OSNR penalty. More specifically, at $30^{\circ}$ phase imbalance, the OSNR penalty after compensation is increased to $1.5 \mathrm{~dB}$ and $2 \mathrm{~dB}$ for case 1 and case 2, respectively, whereas the signal constellation cannot be recovered without compensation. The BASS method also shows a better compensation than the GSOP method, i.e. at a $30^{\circ}$ phase imbalance, the OSNR penalty of the BASS method 
is reduced by $0.4 \mathrm{~dB}$ compared to that of the GSOP method, confirming the effectiveness of the proposed method.

The proposed method is further validated experimentally with 10-Gbaud 4-QAM and 16-QAM signals in the following study.

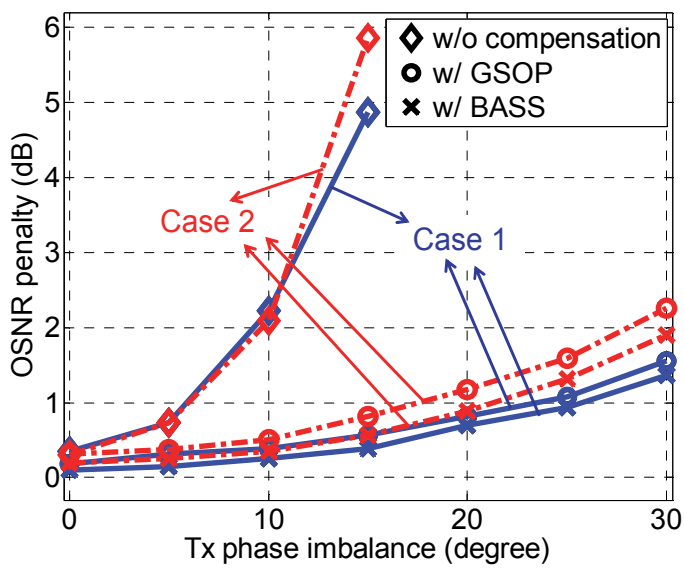

Fig. 3. OSNR penalty of 16-QAM versus Tx phase imbalance. Solid lines: case 1 (B2B); Dash-dotted lines: case 2 (200 km SSMF).

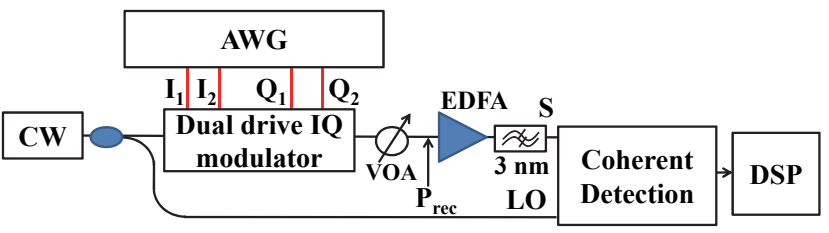

Fig. 4. Experimental setup.

\section{EXPERIMENTAL INVESTIGATION}

In the next step, the Tx IQ imbalance impact is experimentally investigated for 10-Gbaud 4-QAM and 16-QAM optical coherent systems in the back to back case. To simplify the setup in Fig. 1, the same laser is used at the Tx and as local oscillator (LO) at the Rx, as illustrated in Fig. 4. The estimated linewidth of this optical source is about $100 \mathrm{kHz}$. At the Tx, the 4-QAM and 16-QAM signals are generated in the same way as in simulations with an arbitrary waveform generator (AWG). However, its bandwidth is only $4.8 \mathrm{GHz}$, which results in inter-symbol interference (ISI) for 10-Gbaud signals. Fortunately, the ISI effect can also be eliminated simultaneously with the other impairments (i.e. CD) by the equalizer. Note that the Tx phase imbalance is adjusted by modifying the bias voltage controlling the phase difference between the 2 arms of the IQ modulator. Moreover, the signal OSNR is varied by using a variable optical attenuator (VOA) cascaded with an erbium-doped fiber amplifier (EDFA) and followed by a 3-nm optical band-pass filter. At the Rx side, an optical $90^{\circ}$ hybrid splits and crosscombines the $M-\mathrm{QAM}$ signal and the LO. The I and Q components are detected by balanced photodiodes and acquired by a real time oscilloscope with electrical bandwidth of $16 \mathrm{GHz}$ at a sampling rate of $40 \mathrm{GS} / \mathrm{s}$. After data acquisition, DSP is performed offline using the Matlab environment. To emulate the $\mathrm{CFO}$ and $\mathrm{CD}$ impacts on the IQ imbalance compensation, a CFO of $0.2 \mathrm{GHz}$ and a $200-\mathrm{km}$ transmission induced $\mathrm{CD}$ are artificially added to the received samples. BER is measured over 1 million samples. Note that, DSP is applied to blocks of 200000 symbols.
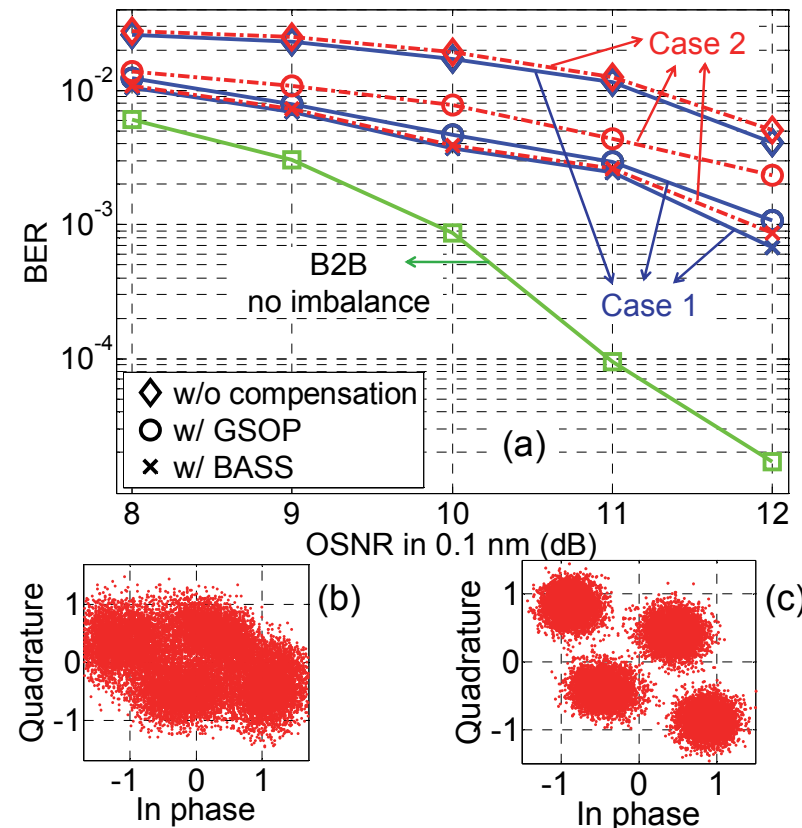

(c)
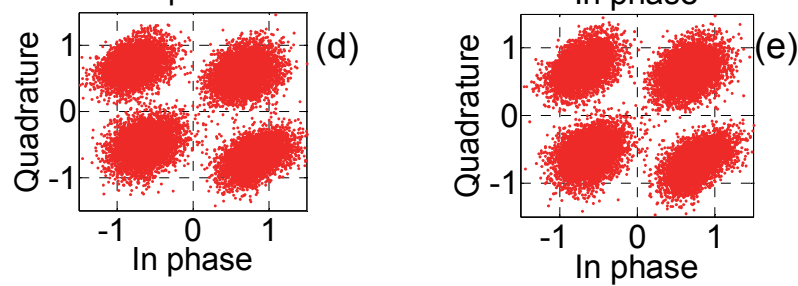

Fig. 5. (a) Evolution of the BER of a 10-Gbaud 4-QAM signal as a function of OSNR in the presence of $30^{\circ}$ phase imbalance (Solid lines: case 1; dash-dotted lines: case 2). Examples of 4-QAM constellations at 10-dB OSNR in the cases of (b) Raw data; (c) w/o IQ imbalance compensation; (d) w/ GSOP (case 2); (e) w/ BASS (case 2).

Fig. 5(a) shows the BER evolution of 4-QAM signals measured in cases 1 and 2 as a function of the OSNR (noise power specified in a $0.1 \mathrm{~nm}$ bandwidth) at $30^{\circ}$ phase imbalance. Note that the phase imbalance value is estimated based on the method in [18]. In case 1, GSOP and BASS exhibit OSNR penalties of $2 \mathrm{~dB}$ and $1.7 \mathrm{~dB}$ at a BER of $10^{-3}$, respectively, compared to the case when no IQ imbalance is present. These penalties are about $1 \mathrm{~dB}$ larger than those obtained in the numerical studies, because several sources of impairments that are possibly present in the experiments (such as the limited AWG bandwidth, etc.) are neglected in the simulation. In the presence of the emulated CFO and CD (case 2), the effectiveness of the GSOP method is reduced by the residual $\mathrm{CFO}$ and $\mathrm{CD}$, whereas the BASS method still presents a good IQ imbalance compensation, even though the $\mathrm{CFO}$ and CD are not completely compensated for. This is due to the independent operation of BASS in a frequency-selective 
channel [14]. As a consequence, the BER curve of the BASS method in case 2 can be made nearly identical to that in case 1 . The BER curve of the GSOP method in case 2 exhibits an OSNR penalty of $1 \mathrm{~dB}$ at a BER of $2 \times 10^{-3}$, compared to that in case 1 . Note that, in case 2 , the ISI generated by the limited AWG bandwidth dominates that due to $\mathrm{CD}$ after the emulated $200-\mathrm{km}$ fiber transmission, explaining the slight degradation of BER curves (w/o IQ imbalance compensation) compared to that in case 1 .

Fig. 5(b) shows an example of a 4-QAM constellation based on the raw data at 10-dB OSNR. Large ISI, caused by the limited AWG bandwidth, is clearly visible. Without IQ imbalance compensation, the equalizer can effectively compensate for this ISI, as shown in Fig. 5(c). However, the BER is sharply degraded if the IQ imbalance is not compensated. Fig. 5(d) and (e) present examples of constellations following compensation by the GSOP and BASS methods, respectively. Even though the IQ imbalance is compensated for, the constellation cannot be fully recovered; indeed the samples distributions change from circles to ellipses after IQ compensation, resulting in penalty as observed in Fig. 5. Note that residual CFO makes the GSOP method less efficient than the BASS method.
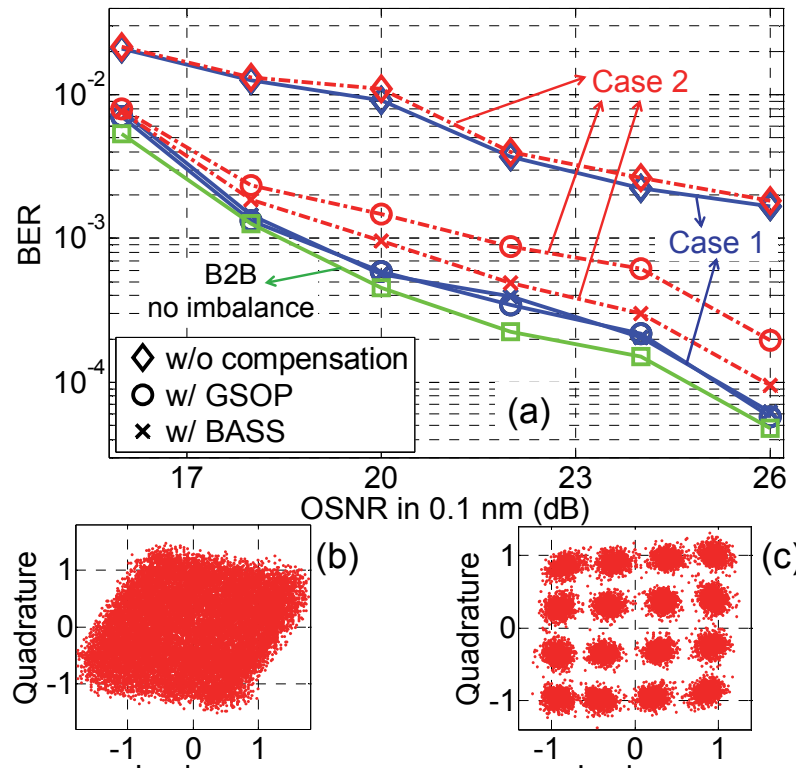

(b)
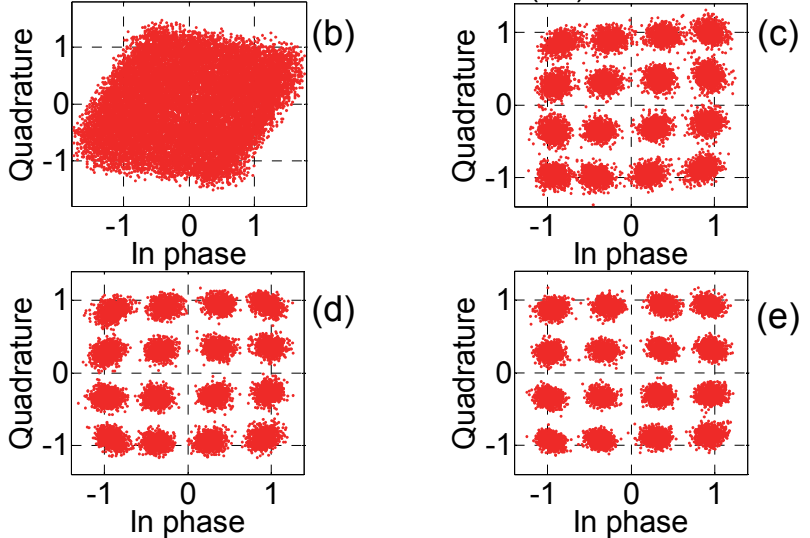

Fig. 6. (a) Evolution of the BER of a 10-Gbaud 16-QAM signal as a function of OSNR in the presence of $10^{\circ}$ phase imbalance (Solid lines: case 1; dash-dotted lines: case 2). Examples of 16-QAM constellations at a 26-dB OSNR in the cases of (b) Raw data; (c) w/o IQ imbalance compensation; (d) w/ GSOP (case 2); (e) w/ BASS (case 2).
In the next step, we validate our algorithm with 16-QAM signals with a $10^{\circ}$ phase imbalance. The reported phase imbalance value is estimated based on the method in [19]. Fig. 6(a) shows the measured BER evolution as a function of OSNR in both cases 1 and 2. As for 4-QAM signals, the B2B curve w/o IQ imbalance is firstly measured as a benchmark. Then the IQ modulator phase-bias is tuned to generate the $10^{\circ}$ phase imbalance. In case 1 , the OSNR penalty is $7 \mathrm{~dB}$ at a BER of $10^{-3}$ if IQ imbalance is not compensated. Thanks to the compensation by the GSOP and BASS methods, the BER curves are brought back to the same level as the BER curve w/o IQ imbalance. Note that the 16-QAM signals are severely distorted by ISI due to the limited AWG bandwidth, as shown in Fig. 6(b) for an OSNR of $26 \mathrm{~dB}$. The equalizer operates effectively to compensate for this ISI, even if the IQ imbalance is not compensated for, leading to a better constellation as demonstrated in Fig. 6(c) for a 26-dB OSNR. In case 2 , where the effects of $\mathrm{CFO}$ and $\mathrm{CD}$ are inserted to raw data, the BER curve w/o IQ imbalance compensation is slightly degraded. As the order of the constellation is increased compared to the 4-QAM case, the effect of residual CFO on the operation of the other DSP stages becomes more important. More specifically, the GSOP decreases its performance, resulting in a 3-dB OSNR penalty at a BER of $10^{-3}$ compared to the case without CFO. Fig. 6(d) presents an example of a 16-QAM constellation at an OSNR of $26 \mathrm{~dB}$, compensated by GSOP. The residual CFO has a reduced influence on the operation of the BASS method, with only 1-dB OSNR penalty at a BER of $10^{-3}$. Fig. 6(e) shows a 16-QAM constellation for a 26-dB OSNR, compensated by the BASS method, showing less dispersed constellation spots compared to those in Fig. 6(d).

\section{HARDWARE COMPLEXITY COMPARISON}

To perform a fair comparison, only the IQ imbalance compensator complexity is compared between the GSOP and BASS methods, regardless of the complexity of the equalizer and other DSP blocks. It is assumed that the same total number of samples, $N$, is used for the different IQ imbalance compensators. Note that, a multiplication between two complex numbers consists of 4 real multiplications and 2 real additions and that squaring a complex number needs 2 real multipliers and 1 real adder. Based on this analysis, Table I provides the summarized hardware complexities of the GSOP and BASS methods in terms of number of real adders, real multipliers and square-root operators. It can be observed that the required total number of multiplier and adder operators of the GSOP method is nearly 3.5 times less than that of the BASS method. However, the BASS method does not require the square-root operator as is the case in the GSOP method, bringing a simpler hardware implementation.

TABLE I. COMPARISON OF HARDWARE COMPELEXITY

\begin{tabular}{|c|c|c|c|}
\hline Methods & Real adders & Real multipliers & $\begin{array}{c}\text { Square-root } \\
\text { operators }\end{array}$ \\
\hline GSOP & $4 N-3$ & $6 N+4$ & 2 \\
\hline BASS & $13 N$ & $22 N$ & 0 \\
\hline
\end{tabular}

$N$ - total number of samples used for IQ imbalance compensation 
It should furthermore be noted that our proposed method operates at the symbol rate, leading to a promising approach for parallel processing (which is preferable at the high bit-rates used in optical transmission systems), whereas the GSOP method uses statistical calculation that can create a delay in parallel implementations.

\section{CONCLUSION}

In this paper, we have studied and demonstrated a promising method for IQ imbalance compensation based on blind adaptive source separation in optical $M-\mathrm{QAM}$ coherent communication systems. The proposed method is numerically and experimentally validated with 10 -Gbaud optical 4-QAM and 16-QAM signals subjected to $30^{\circ}$ and $10^{\circ}$ transmitter phase imbalance. The impacts of carrier frequency offset and chromatic dispersion are also emulated to verify the robustness of the proposed method. In the presence of such impairments, it is found that the CFO should be compensated for before the IQ imbalance to achieve a better performance. Compared to the Gram-Schmidt orthogonalization procedure, the BASS method outperforms the GSOP method in the presence of residual CFO and CD. Although our proposed method requires more adder and multiplier operators compared to the GSOP, it can operate at the sample rate, which is highly suitable for parallel implementation.

\section{ACKNOWLEDGMENT}

This work was financed by the Contrat de Plan EtatRégion PONANT and the French Ministry of Research.

\section{REFERENCES}

[1] P. C. Schindler, D. Korn, C. Stamatiadis, M. F. O'Keefe, L. Stampoulidis, R. Schmogrow, P. Zakynthinos, R. Palmer, N. Cameron, Y. Zhou, R. G. Walker, E. Kehayas, S. Ben-Ezra, I. Tomkos, L. Zimmermann, K. Petermann, W. Freude, C. Koss, and J. Leuthold, "Monolithic GaAs electro-optic IQ modulator demonstrated at 150 Gbit/s with 64QAM," J. Lightw. Technol., vol. 32, no. 4, pp. 760-765, Feb. 2014.

[2] I. Fatadin, S. J. Savory, and D. Ives, "Compensation of quadrature imbalance in an optical QPSK coherent receiver," IEEE Photon. Technol. Lett., vol. 20, no. 20, pp. 1733-1735, Oct. 2008.

[3] R. Rios-Muller, J. Renaudier, and G. Charlet, "Blind receiver skew compensation and estimation for long-haul non-dispersion managed systems using adaptive equalizer," J. Lightw. Technol., vol. 33, no. 7, pp. 1315-1318, Apr. 2015.

[4] T. Koike-Akino, D. S. Millar, K. Kojima, K. Parsons, T. Yoshida, K. Ishida, Y. Miyata, W. Matsumoto, and T. Mizuochi, "Turbo demodulation for LDPC-coded high-order QAM in presence of transmitter angular skew," in Proc. ECOC 2014, Cannes, France, paper Th.1.3.2.

[5] G. Khanna, S. Calabro, B. Spinnler, E. de Man, and N. Hanik, "Joint adaptive pre-compensation of transmitter $\mathrm{I} / \mathrm{Q}$ skew and frequency response for high order modulation formats and high baud rates," in Proc. OFC 2015, Los Angeles, CA, USA, paper M2G.4.

[6] J.-F. Cardoso, and B. H. Laheld., "Equivariant adaptive source separation," IEEE Trans. Signal Processing, vol. 44, no. 12, pp. 30173030, Dec. 1996

[7] C. Rottondi, M. Tornatore, A. Pattavina, and G. Gavioli, "Routing, modulation level, and spectrum assignment in optical metro ring networks using elastic transceivers," J. Opt. Commun. Netw., vol. 5, no. 4, pp. 305-315, Apr. 2013.

[8] P. J. Winzer, A. H. Gnauck, C. R. Doerr, M. Magarini, and L. L. Buhl, "Spectrally efficient long-haul optical networking using 112-Gb/s polarization-multiplexed 16-QAM," J. Lightw. Technol., vol. 28, no. 4 pp. 547-556, Feb. 2010.

[9] T. Schenk, RF imperfections in high-rate wireless systems, 1st ed. Springer Netherlands, 2008, pp. 143-144.

[10] S. J. Savory, "Digital filters for coherent optical receivers," Opt. Express, vol. 16, no. 2, pp. 804-817, Jan. 2008.

[11] M. Selmi, Y. Jaouen, and P. Ciblat, "Accuarate digital frequency offset estimator for coherent PolMux QAM transmission systems," in Proc. ECOC 2009, Vienna, Austria, paper P3.08.

[12] A. J. Viterbi, and A. M. Viterbi, "Nonlinear estimation of PSKmodulated carrier phase with application to burst digital transmission," IEEE Trans. Inform. Theory, vol. IT-29, no. 4, Jul. 1983.

[13] S.-I. Amari, and J.-F. Cardoso, "Blind source separation Semiparametric statistical approach," IEEE Trans. Signal Processing, vol. 45, no. 11, pp. 2692-2700, Nov. 1997

[14] M. Valkama, M. Renfors, and V. Koivunen, "Blind signal estimation in conjugate signal models with application to $\mathrm{I} / \mathrm{Q}$ imbalance compensation,” IEEE Signal Process. Lett., vol. 12, no. 11, pp. 733-736, Nov. 2005.

[15] M. S. Faruk, and K. Kikuchi, "Compensation for in-phase/quadrature imbalance in coherent receiver front-end for optical quadrature amplitude modulation," IEEE Photon. J., vol. 5, no. 2, article no. 7800110, Apr. 2013.

[16] B. Razavi, "Design considerations for direct-conversion receivers," IEEE Trans. Circuits Syst. II, Analog Digit. Signal Process., vol. 44, no. 6, pp. 428-435, Jun. 1997.

[17] Characteristics of single-mode optical fibre and cable, International Telecommunications Union ITU-T Recommendation G.652, Mar. 2003.

[18] T.-H. Nguyen, F. Gomez-Agis, M. Gay, L. Anet-Neto, P. Scalart, C. Peucheret, M. Joindot, O. Sentieys, J.-C. Simon, and L. Bramerie, "IQ imbalance compensation based on maximum SNR estimation in coherent QPSK systems," in Proc. ICTON 2014, Graz, Austria, paper Tu.C1.3.

[19] T.-H. Nguyen, P. Scalart, M. Joindot, M. Gay, L. Bramerie, C. Peucheret, A. Carer, J.-C. Simon, and O. Sentieys, "Joint simple blind IQ imbalance compensation and adaptive equalization for 16-QAM optical communications," in Proc. ICC 2015, London, UK, pp. 49134918. 